\title{
Rectification and Flux Reversals for Vortices Interacting with Triangular Traps
}

\author{
C.J. Olson Reichhardt and C. Reichhardt \\ Center for Nonlinear Studies and Theoretical Division, Los Alamos National Laboratory, Los Alamos, New Mexico 87545
}

(October 31, 2018)

\begin{abstract}
We simulate vortices in superconductors interacting with two-dimensional arrays of triangular traps. We find that, upon application of an ac drive, a net dc flow can occur which shows current reversals with increasing ac drive amplitude for certain vortex densities, in agreement with recent experiments and theoretical predictions. We identify the vortex dynamics responsible for the different rectification regimes. We also predict the occurrence of a novel transverse rectification effect in which a dc flow appears that is transverse to the direction of the applied ac drive.

PACS numbers: 74.25.Qt
\end{abstract}

Vortices interacting with periodic pinning structures have been attracting considerable attention since they are an ideal system in which to study the static and dynamical behaviors of collectively interacting particles coupled to periodic substrates. In these systems, a variety of commensurability effects and novel vortex crystals can occur as a function of the ratio of the number of vortices to the number of pinning sites [1-3]. The vortex crystals are composed of one or more vortices trapped at each pin with any remaining vortices located in the interstitial regions between the pinning sites. A variety of dynamical flow phases occur when a drive is applied, including the flow of interstitial vortices between the pinning sites $[4,5]$ as well as channeling along rows of pinning [4]. Much of the physics of vortices interacting with periodic pinning is also observed for repulsively interacting colloids in periodic optical trap arrays [6-8]. In addition to the basic science issues, vortices interacting with periodic pinning arrays are relevant to possible technological applications of superconductors, such as critical current enhancement or controlled motion of flux for new types of devices.

Several methods have been proposed for using periodic pinning or controlled disorder in superconductors to create vortex ratchets, rectifiers, and logic devices [9-13]. As in general ratchet systems, which can be deterministic or stochastic in nature [14], a vortex ratchet transforms an ac input into a dc response. Vortex ratchets can be created via asymmetric periodic pinning lines [9,10], asymmetric channels, or the asymmetry introduced by multiple ac drives in a system with a symmetric substrate $[11,12]$. Ratchets constructed of periodic twodimensional (2D) arrays of asymmetric pinning sites have also been proposed [13]. Recently, both positive and negative vortex rectification have been experimentally realized in periodic arrays of triangular pinning sites [15]. In Ref. [15], the triangles are arranged in a square lattice with the tips of the triangles oriented in the $+y$ direction. When an ac force is applied in the $y$ direction at low matching fields, rectification of the vortex motion in the $+y$ direction occurs over a range of ac amplitudes, with a peak rectification at a particular amplitude. For higher matching fields, there is a $-y$ rectification at lower ac drives, followed by a $+y$ rectification at higher ac drives.
This change in the rectification direction is explained in Ref. [15] as originating from the separate motion of interstitial vortices at low drives, giving $-y$ rectification, and the motion of the pinned vortices at higher drives, producing $+y$ rectification. It is not clear how the motions of these two vortex species can be fully separated, especially in the presence of thermal fluctuations, and thus a clearer picture of the vortex dynamics producing the rectification is needed.

In this work we present simulations of vortices interacting with $2 \mathrm{D}$ square arrays of triangular pinning sites for parameters corresponding to the recent experiments of Ref. [15]. We find a $+y$ rectification at low ratios $n$ of the number of vortices to the number of pinning sites, with a maximum $+y$ dc flux as a function of ac amplitude. When interstitial vortices are present, the initial rectification is in the $-y$ direction, and is followed by a crossover to $+y$ rectification at higher ac amplitude, as seen in experiments. We find that for large ac amplitudes, when the motion of the vortex lattice becomes elastic, the rectification switches to the $-y$ direction, which is explained in terms of channeling effects. We also predict a novel transverse ratchet effect where a dc motion is generated in the direction transverse to the applied ac drive. All of the effects we describe here should also occur for repulsively interacting colloidal particles interacting with $2 \mathrm{D}$ triangular traps.

We consider a thin film superconductor containing a square array of $N_{p}=64$ triangular pinning sites with periodic boundary conditions in the $x$ and $y$-directions. We add $N_{v}=n N_{p}$ vortices to the sample, each of which obeys the overdamped equation of motion

$$
\eta \frac{d \mathbf{r}_{i}}{d t}=\mathbf{f}_{i}^{v v}+\mathbf{f}_{p}+\mathbf{f}_{A C}+\mathbf{f}_{T} .
$$

Here the vortex-vortex interaction force, appropriate for a thin film superconductor, is $\mathbf{f}_{i}^{v v}=$ $\sum_{j \neq i}^{N_{v}}\left(\Phi_{0}^{2} / \mu_{0} \pi \Lambda\right) \hat{r} / r_{i j}$, where $\Phi_{0}$ is the elementary flux quantum and $\Lambda$ is the thin film screening length [16]. We use a fast summation method to evaluate this long-range interaction [17]. The pinning force $\mathbf{f}_{p}$ comes from a square array of equilateral triangles with one vertex pointing in the $+y$ direction, as shown in Fig. 1. Every triangular 

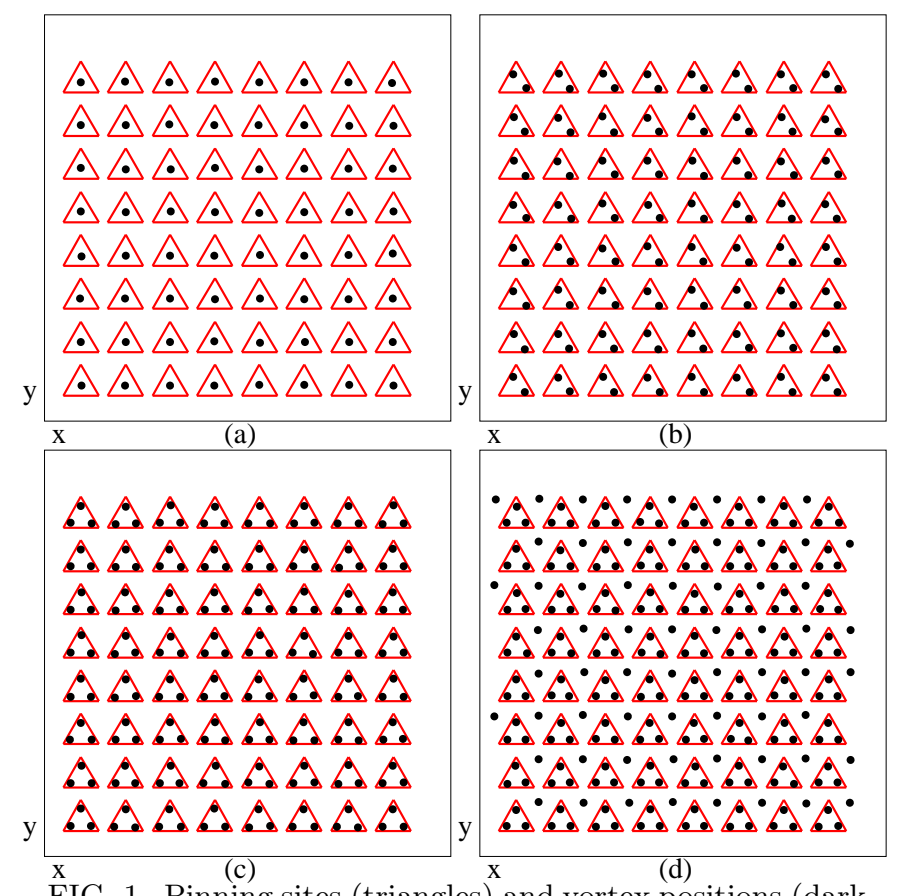

FIG. 1. Pinning sites (triangles) and vortex positions (dark circles) at the matching fields $n=$ (a) 1, (b) 2, (c) 3, and (d) 4.

pin is modeled as three half-parabolas of equal strength, each of which attracts the vortex to a line passing through the center of the triangle and parallel to one of the sides. The pinning force is cut off at the edge of the triangle. The applied ac force $\mathbf{f}_{A C}=A \sin (\omega t) \hat{\mathbf{r}}$ acts in the direction perpendicular to an applied ac current. Here we consider either $f_{A C}^{y}=A \sin (\omega t) \hat{\mathbf{y}}$ or $f_{A C}^{x}=A \sin (\omega t) \hat{\mathbf{x}}$, with no mixtures of ac drives. Temperature is modeled as random thermal kicks with the property $\left\langle\mathbf{f}_{T}(t)>=0\right.$ and $<\mathbf{f}_{T}(t) \mathbf{f}_{T}\left(t^{\prime}\right)>=2 \eta k_{B} T \delta\left(t-t^{\prime}\right)$.

We match our parameters to those of the experiment in Ref. [15], performed in Nb films with $\eta=1.4 \times 10^{-12}$ $\mathrm{Ns} / \mathrm{m}$. We take $T / T_{c}=0.98$, giving $f_{T}=0.46 f_{0}$, where $f_{0}=\phi_{0}^{2} / 2 \pi \mu_{0} \lambda^{3}=1.09 \times 10^{-5} \mathrm{~N} / \mathrm{m}$. At this temperature, the London penetration depth $\lambda=\lambda(0) /(1-$ $\left.\left(T / T_{c}\right)^{2}\right)^{1 / 2}=368 \mathrm{~nm}$, so our pin spacing is $2.09 \lambda$ in the $x$ direction and $2.03 \lambda$ in the $y$ direction, and the pins are $1.68 \lambda$ on a side. In the experiment, each pin held a maximum of three vortices, so to match this we set the pinning strength to $f_{p}=1.05 f_{0}$. We fix $\omega=78 \mathrm{kHz}$, higher than experiment due to the limitation of simulation time; however, since the behavior of the system is controlled by the ratio $A / \omega$, we compensate by taking $A$ larger than in experiment. We note that we find the same generic behaviors for other parameters, including a pinning array that is triangular rather than square.

In Fig. 1 we show the vortex positions and pin locations at $A=0$ for a system with a square array of triangular pins at fillings $n=1,2,3$, and 4. A global symmetry breaking occurs at $n=2$ between the two possible arrangements of the vortices allowed by the square pinning
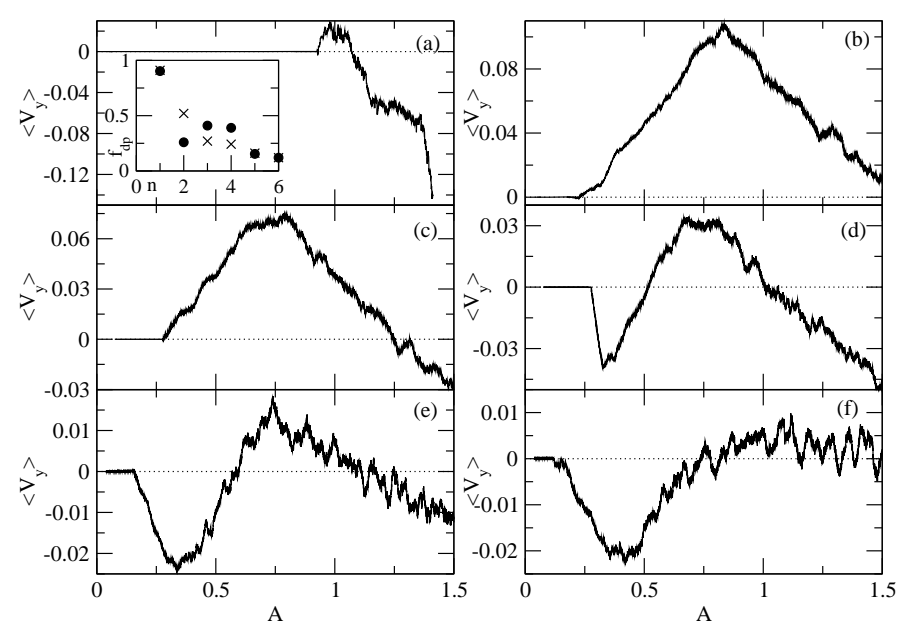

FIG. 2. Net dc velocity $\left\langle V_{y}>\right.$ in units of $\mathrm{m} / \mathrm{s}$ as a function of applied ac current $A$ in units of $f_{0}$ for $n=$ (a) 1, (b) 2, (c) 3 , (d) 4 , (e) 5 , and (f) 6 . Inset to (a): dc depinning force $\left|f_{d p}\right|$ for $+y$ (circles) and $-y$ (x's) directions.

lattice. All of the vortices sit in the pins for $n \leq 3$, but for $n \geq 4$ the vortex-vortex interaction is strong enough that some vortices move to the interstitial regions.

We first consider the dc depinning force $f_{d p}$ in the positive and negative $y$ directions by applying a dc driving force of increasing magnitude and measuring the average vortex velocity $\left\langle V_{y}\right\rangle$. In the inset to Fig. 2(a) we plot $\left|f_{d p}\right|$ as a function of $n$. At $n=1$ the depinning is symmetric in the $\pm y$ directions, as expected given our model for the pinning. For $n=2,\left|f_{d p}^{-y}\right|$ is twice as large as $\left|f_{d p}^{+y}\right|$, due to the fact that the vortices align vertically for $+y$ driving, and horizontally for $-y$ driving. For $n=3$ and $4,\left|f_{d p}^{+y}\right|$ is $25 \%$ larger than $\left|f_{d p}^{-y}\right|$. Here, the vortex at the top of each pin acts to assist in the depinning of the vortices along the bottom of the pin for $-y$ driving. For $n>4$, the depinning is dominated by the interstitial vortices and the asymmetry in the depinning is lost. The depinning asymmetry at $n=2$ to 4 should be large enough to be observable experimentally. Depinning in the $\pm x$ directions is symmetric for all $n$. Asymmetric depinning at noninteger $n$ was observed in Ref. [18].

We next consider the effect of an applied ac drive $f_{A C}^{y}$. We monitor the net dc velocity $\left\langle V_{y}\right\rangle$ at fixed $\omega$ and sweep the ac amplitude $A$, averaging $\left\langle V_{y}\right\rangle$ for 20 periods at each increment of $A$. In Fig. 2(a) we plot $\left\langle V_{y}\right\rangle$ vs $A$ for $n=1$. For $A<0.9$, there is no net flow in $x$ or $y$, indicating that the vortices are still pinned. For $A=0.9$ a finite $+y$ dc velocity appears with no net $x$ dc velocity, corresponding to a $+y$ rectification. As $A$ increases further, $\left\langle V_{y}\right\rangle$ increases, reaching a maximum at $A=0.99$ and then dropping back to zero at $A=1.05$. This behavior matches that seen in the experiments of Ref. [15]. We find no steps in the $+y$ rectification regime, in agreement with the experiments, but in disagreement with recent $T=0$ simulations of a very small system for asymmetric pinning sites that predicted steps in the velocity vs 

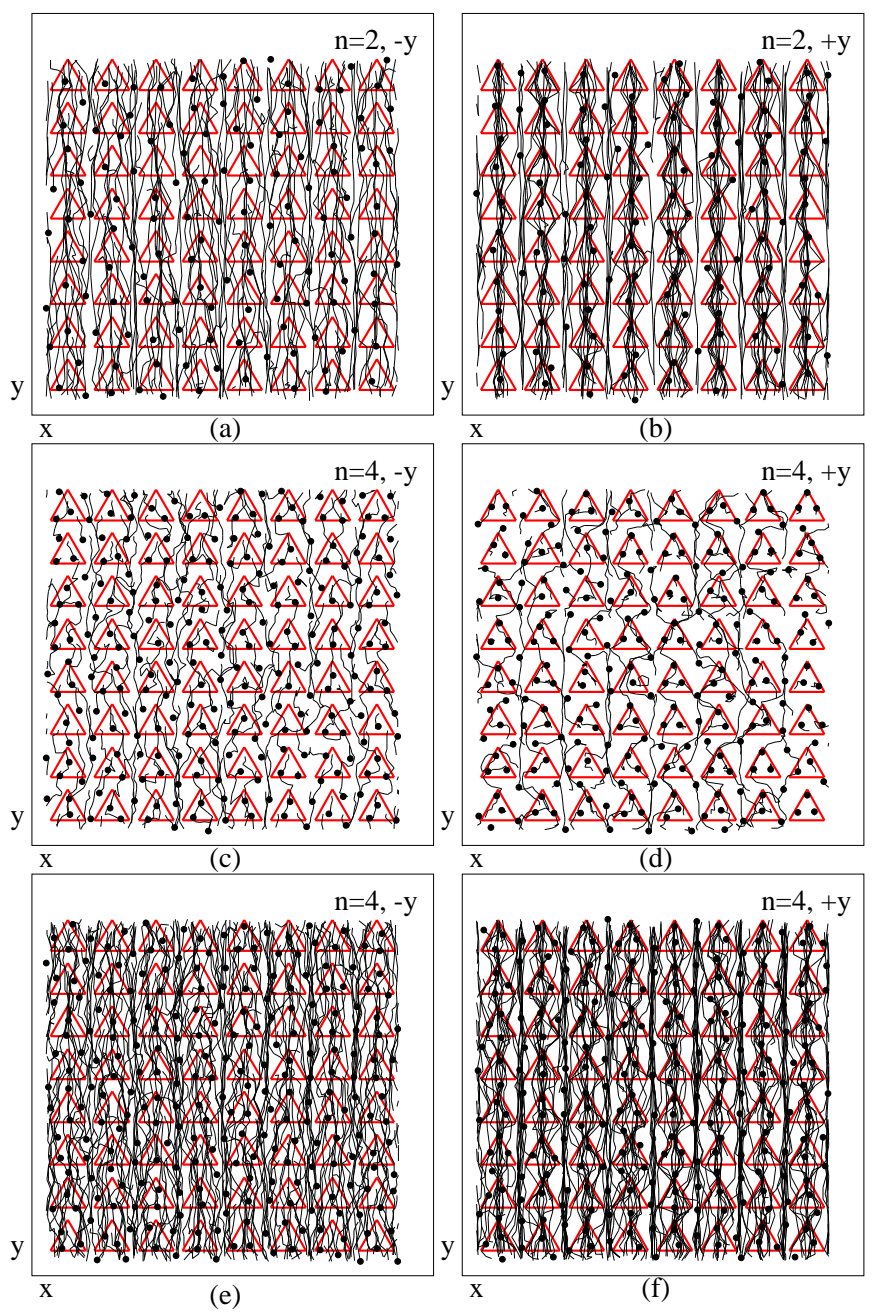

FIG. 3. Pins (triangles), vortices (dots), and vortex trajectories during $1 / 4$ of the driving period (lines) for: (a) $N=2$, $A=1.0,-y$ portion of drive cycle, (b) $+y$ portion of same cycle; (c) $N=4, A=0.35,-y$ portion of drive cycle, (d) $+y$ portion of same cycle; (e) $N=4, A=0.75,-y$ portion of drive cycle, (f) $+y$ portion of same cycle.

$f_{A C}$ curve [19]. The transport does not occur in an organized step like flow, but is instead a stochastic process, as we will show in more detail in Fig. 3. In Fig. 2(b) and Fig. 2(c) we plot $\left\langle V_{y}\right\rangle$ for $n=2$ and 3, respectively. These curves are similar to the $n=1$ case, with an initial pinned phase followed by $+y$ rectification that goes through a maximum with increasing $A$.

In Fig. 2(d) we show $<V_{y}>$ for the case of $n=4$ when interstitial vortices are present, as seen in Fig. 1(d). Here there is an initial negative rectification regime in the $-y$ direction, followed by a crossover to a $+y$ rectification as seen in experiments [15]. In Fig. 2(e,f) we plot the cases for $n=5$ and $n=6$, respectively, which show a similar rectification to that in Fig. 2(d). In all of these cases, a combination of interstitial and pinned vortices are present. As $n$ is increased further, the ratcheting effects are gradually reduced and become indistinguishable from thermal noise.

For $n=1$ in Fig. 2(a), at higher $A>1.05,<V_{y}>$ becomes negative, indicating $-y$ rectification at high drives. This reversal occurs for all fillings but is most pronounced for $n=1$ and $n=4$, when the onset of the reversal falls at lower values of $A$. We predict that this effect should be easily observable in experiment, although it was not reported in Ref. [15]. The high-drive $-y$ rectification originates when the $-y$ vortex motion occurs through interstitial channels while the $+y$ vortex motion becomes localized on top of a column of pinning, giving a larger drag during the $+y$ portion of the cycle.

To understand the role of interstitial and pinned vortices in the rectification process, we examine the dynamics of the vortices. In Fig. 3(a,b) we illustrate a typical example of vortex motion leading to $+y$ rectification at $n=2$ and $A=1.0$. Fig. 3(a) shows the vortex trajectories during $1 / 4$ of the drive cycle when the drive is in the $-y$ direction. At these temperatures, none of the vortices remain pinned. The vortex motion is uncoordinated and the vortices wander stochastically as they pass through the pinning. In contrast, during the $+y$ portion of the cycle shown in Fig. 3(b), the vortices are channeled both within and between the columns of pinning sites, and there is little wandering in the $x$ direction. This more focused motion leads to a greater overall displacement in the $+y$ direction, and a net $+y$ rectification. Similar motion occurs for $n=1$ and $n=3$, except that for $n=1$ all of the motion is confined to the pinning channel and there is no interstitial motion, so the net rectification is correspondingly reduced as seen in Fig. 2(a).

At $n=4$ and above, when interstitial vortices are present, $-y$ rectification occurs at low drives, as shown in Fig. 2(d-f). In Fig. 3(c-d) we illustrate an example of vortex motion in the $-y$ rectification regime for $n=4$ and $A=0.35$. Fig. 3(c) shows that the vortices meander moderately in the $x$ direction during the $-y$ portion of the drive cycle. Note that none of the vortices remain pinned; instead, all of the vortices are participating in the motion, with pinned and interstitial vortices switching places frequently. During the $+y$ portion of the drive cycle shown in Fig. 3(d), the drive is too low to overcome the pinning force and allow the formation of channels of the type seen in Fig. 3(b). Instead, the $+y$ vortex flow is strongly diverted into the $x$ direction by the pins, reducing the net motion in the $+y$ direction, and resulting in an overall $-y$ rectification. As $A$ is further increased, the vortices begin to overcome the pinning in the $+y$ direction and form channels during the $+y$ portion of the cycle, as illustrated in Fig. 3(f) for $n=4$ and $A=0.75$. This gives $+y$ rectification just as in Fig. $3(\mathrm{a}-\mathrm{b})$. As $n$ increases above 4 , the channels become more closely packed and the amount of vortex wandering in the $x$ direction during the $-y$ portion of the cycle decreases, progressively lowering the net $+y$ rectification. The onset of the $-y$ and $+y$ rectification phases also drops to 

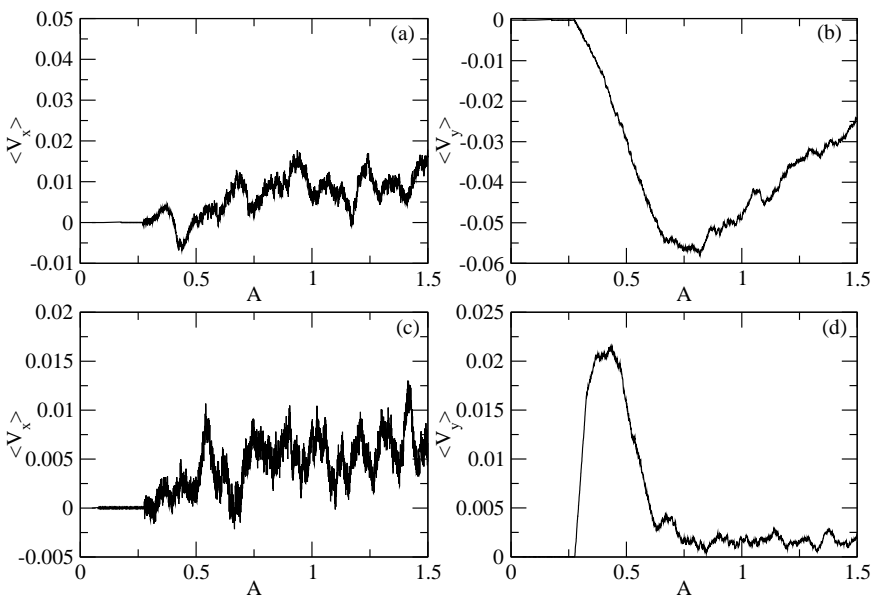

FIG. 4. Transverse rectification for $f_{A C}^{x}$. (a) $\left\langle V_{x}>\right.$ for $n=2$; (b) corresponding $\left\langle V_{y}\right\rangle$ with $-y$ rectification. (c) $<V_{x}>$ for $n=4$; (b) corresponding $<V_{y}>$ with $+y$ rectification.

lower $A$ with increasing $n$ since the effectiveness of the pinning decreases with increasing vortex density.

In Fig. 4 we illustrate $\left\langle V_{x}\right\rangle$ and $\left\langle V_{y}\right\rangle$ when an ac drive $f_{A C}^{x}$ is applied in the $x$ direction for $n=2$ and 4. The net $x$ velocity is zero but we find a transverse rectification in the $y$ direction: $-y$ for $n=2$ and $+y$ for $n=4$. The magnitude of the rectification is comparable to that seen for $f_{A C}^{y}$, and it should thus be experimentally observable. The transverse ratchet effect is produced by the interaction of the vortices with the pinning sites. For $n=2$, the vortices channel along the rows of pins and are deflected downward by the pinning sites, producing $-y$ rectification. Similar motion occurs for $n=3$. For $n=4$ to 6 , the interstitial vortices dominate the motion through channels that pass along the tips of the triangles, although all vortices are being pinned and depinned. The interstitial vortices are deflected upward by the vortices inside the pins, producing $+y$ rectification. Since the transverse motion does not require breaking of the $x$ direction symmetry of the system, it can also be observed for a dc $x$ direction drive.

In conclusion, we have conducted simulations of vortices in thin film superconductors interacting with triangular pinning sites for parameters relevant to recent experiments. We show that both $\mathrm{a}+y$ and $-y$ rectification can occur at depinning depending on whether interstitial vortices are present, in agreement with experiment. We find dc depinning anisotropy for $n=2$ to 4 , but observe that even when the dc depinning is not anisotropic, rectification can still occur due to the fact that the vortex dynamics differs under ac and dc drives. Rectification in the $+y$ direction occurs when the ac drive overcomes the pinning strength and vortex channels form that flow in the $+y$ direction. When interstitial vortices are present at zero ac drive, an initial $-y$ rectification occurs at lower ac drives due to scattering of the $+y$ vortex motion by the pinning sites. This occurs for $n=4$ and above, in agree- ment with experiments. We also predict that a novel transverse ratchet effect should occur when the ac drive is applied in the $x$ direction. Here, the rectification is in the $y$ direction. Our results should be generic to any type of repulsively interacting particles moving through triangular traps and may have potential applications for species fractionalization in colloidal systems.

This work was supported by the U.S. DoE under Contract No. W-7405-ENG-36.

[1] M. Baert et al., Phys. Rev. Lett. 74, 3269 (1995); K. Harada et al., Science 274, 1167 (1996); V. Metlushko et al., Phys. Rev. B 59, 603 (1999); S.B. Field et al., Phys. Rev. Lett. 88, 067003 (2002); A.N. Grigorenko et al., Phys. Rev. Lett. 90, 237001 (2003).

[2] C. Reichhardt, C.J. Olson, and F. Nori, Phys. Rev. B 57, 7937 (1998); C. Reichhardt and N. Grønbech-Jensen, Phys. Rev. Lett. 85, 2372 (2000); C. Reichhardt et al., Phys. Rev. B 64, 052503 (2001).

[3] J.I. Martín et al., Phys. Rev. Lett. 79, 1929 (1997); D.J. Morgan and J.B. Ketterson, ibid. 80, 3614 (1998); J.I. Martín et al., ibid. 83, 1022 (1999).

[4] C. Reichhardt, C.J. Olson, and F. Nori, Phys. Rev. Lett. 78, 2648 (1997); B.Y. Zhu et al., Phys. Rev. B 64, 012504 (2001); C. Reichhardt, G.T. Zimányi, and N. GrønbechJensen, Phys. Rev. B 64, 014501 (2001).

[5] L. Van Look et al., Phys. Rev. B 60, R6998 (1999); C. Reichhardt et al., Phys. Rev. B 61, R11914 (2000).

[6] P.T. Korda, G.C. Spalding, and D.G. Grier, Phys. Rev. B 66, 024504 (2002); P.T. Korda, M.B. Taylor, and D.G. Grier, Phys. Rev. Lett. 89, 128301 (2002); K. Ladavac, K. Kasza, and D.G. Grier, cond-mat/0310396; M.P. MacDonald, G.C. Spalding, and K. Dholakia, Nature (London) 426, 421 (2003).

[7] M. Brunner and C. Bechinger, Phys. Rev. Lett. 88, 248302 (2002); C. Reichhardt and C.J. Olson, Phys. Rev. Lett. 88, 248301 (2002).

[8] K. Mangold, P. Leiderer, and C. Bechinger, Phys. Rev. Lett. 90, 158302 (2003).

[9] C.S. Lee, B. Jankó, I. Derenyi, and A.L. Barabasi, Nature (London) 400, 337 (1999).

[10] C.J. Olson, C. Reichhardt, B. Jankó, and F. Nori, Phys. Rev. Lett. 87, 177002 (2001).

[11] C. Reichhardt, C.J. Olson, and M.B. Hastings, Phys. Rev. Lett. 89, 024101 (2002).

[12] M.B. Hastings, C.J. Olson Reichhardt, and C. Reichhardt, Phys. Rev. Lett. 90, 247004 (2003).

[13] B.Y. Zhu, F. Marchesoni, V.V. Moshchalkov, and F. Nori, Phys. Rev. B 68, 014514 (2003).

[14] P. Reimann, Phys. Rep. 361, 57 (2002).

[15] J.E. Villegas et al., Science 302, 1188 (2003).

[16] J.R. Clem, Phys. Rev. B 43, 7837 (1991).

[17] N. Grønbech-Jensen, Comput. Phys. Commun. 119, 115 (1999).

[18] B.Y. Zhu et al., Physica E 18, 322 (2003).

[19] B.Y. Zhu, F. Marchesoni, and F. Nori, Physica E 18, 318 (2003). 